\title{
Estereotipos negativos hacia el envejecimiento en estudiantes universitarios del área de la salud
}

\author{
Lara-Pérez, Ricardo*; Moreno-Pérez, Norma Elvira²; Padilla-Raygoza, Nicolás ${ }^{3}$
}

\section{RESUMEN}

Introducción: Los estereotipos negativos hacia el envejecimiento generan conductas discriminatorias (viejismo) hacia los adultos mayores. Objetivo: Analizar la presencia e intensidad de los estereotipos negativos hacia el envejecimiento presentes entre los estudiantes del área de la salud de una universidad de la región centro de México. Metodología: Estudio cuantitativo, descriptivo, transversal y comparativo; muestreo estratificado de $n=326$ estudiantes inscritos en el período agosto-diciembre. Aplicación del Cuestionario de Evaluación de Estereotipos Negativos hacia la Vejez (CENVE), cuyo puntaje oscila entre 15 y 60 puntos, mayor puntuación indica un elevado grado de estereotipos negativos hacia la vejez. Se utilizó estadística descriptiva para variables categóricas por distribución de frecuencias y porcentajes, y para las numéricas, medidas de tendencia central y dispersión. Resultados: Edad media de 20.15 1.67 años, predomina el género femenino (79.9\%), puntaje $36.24 \pm 4.96$, los estereotipos hacia la vejez que predominaron fueron en la dimensión de carácter-personalidad. Existe presencia de estereotipos negativos en el 37\% de los casos, sin diferencias entre los grupos. La presencia de estereotipos negativos fue mayor en comparación con estudios sudamericanos y europeos. La dimensión que presentó más estereotipos hacia la vejez difiere respecto a estudios internacionales y nacionales donde la que presenta mayor estereotipo es la dimensión salud. Conclusiones: Prevalecen elevados estereotipos negativos en la población de estudio, la dimensión más afectada fue la de carácter-personalidad. Se recomienda la implementación de programas desde la de educación básica, que favorezcan la construcción de habilidades socioemocionales que ayuden a disminuir el viejismo.

Palabras clave: Estereotipo; Envejecimiento; Estudiantes; Ciencias de la Salud (DeCS; BIREME).

1'Licenciado en Enfermería y Obstetricia. Departamento de Enfermería, División de Ciencias de la Salud e Ingenierías. Universidad de Guanajuato. Celaya, Guanajuato, México. Correo Electrónico: drlpr@hotmail.com ORCID ID: orcid.org/0000-0001-8648-7013.

²Doctora en Ciencias de Enfermería. Departamento de Enfermería Clínica, Universidad de Guanajuato. Celaya, Guanajuato, México. Correo Electrónico: normaelvira.moreno2@gmail.com ORCID: 0000-0003-1829-3916.

${ }^{3}$ Doctor en Epidemiología.Departamento de Enfermería y Obstetricia, Universidad de Guanajuato. Celaya, Guanajuato, México.

Recibido: 10/12/2018

Aceptado: 20/12/2018

*Autor para correspondencia

Cómo citar este artículo

Lara-Pérez R, Moreno-Pérez NE, Padilla-Raygoza N. Estereotipos negativos hacia el envejecimiento en estudiantes universitarios del área de la salud. SANUS. 2018; (8): 10-25. [Acceso_____]; Disponible en: mes día año URL 


\section{Estereótipos negativos para o envelhecimento em estudantes universitários da área da saúde}

\section{RESUMO}

Introdução: Estereótipos negativos para o envelhecimento gerar comportamento discriminatório (envelhecida) para os idosos. Objetivo: Analise a presença e intensidade de estereótipos negativos em direção ao envelhecimento presente entre os estudantes da área da saúde da Universidade de Guanajuato UGTO). Metodologia: Estudo quantitativo, descritivo, transversal e comparativo; amostragem estratificada de $n=326$ alunos matriculados no período de agosto a dezembro. Aplicação do Questionário de Avaliação de Estereótipos Negativos para a Velhice (CENVE), cuja pontuação varia entre 15 e 60 pontos; maior pontuação indica um alto grau de estereótipos negativos em relação à idade avançada. Estatísticas descritivas foram utilizadas para variáveis categóricas por distribuição de freqüência e porcentagens, e para o numérico, medidas de tendência central e dispersão. Resultados: Média de idade de 20.15 \pm 1.67 anos; o gênero feminino predomina (79.9\%); pontuar CENVE 36.24 \pm 4.96 ; a dimensão mais afetada foi Character-Personality. Há uma presença de estereótipos negativos em 37\% dos casos, não há diferenças entre os grupos. A presença de estereótipos negativos foi maior em comparação com estudos sul-americanos; Da mesma forma, o escore do CENVE foi superior ao dos estudos realizados na Espanha e na Costa Rica. A dimensão mais afetada difere dos estudos internacionais e nacionais, onde a dimensão mais afetada é a dimensão Saúde. Conclusões: Estereótipos negativos elevados prevalecem na população estudada; a dimensão mais afetada foi Personagem-Personalidade. Recomenda-se a implementação de programas da educação básica, que favorecem a construção de Habilidades sócio-emocionais que ajudam a reduzir a discriminação etária.

Palavras chave: Estereótipos; envelhecimento; estudantes; Ciências da Saúde (DeCS; BIREME).

\section{INTRODUCCIÓN}

En México, la población adulta mayor representa el 10.4\% del total, y a pesar del aumento de la esperanza de vida, no significa que se envejezca con calidad; debido a que esta población se enfrenta a problemas como discapacidades y comorbilidades de carácter crónico-degenerativo. Además de otras carencias resultantes de la falta de una derechohabiencia (16.4\%), y la jubilación (66.4\%), originando de esta manera, un estado de pobreza multidimensional $(43.7 \%)^{(1)}$.

Esta situación genera la aparición de estereotipos negativos hacia la vejez. Y es que existe una construcción social para catalogar a las personas sobre lo que "no son"; y las múltiples pérdidas que sufre el adulto mayor provocan que sea visto como un individuo no productivo, alguien enfermo que debe ser medicado y excluido del sistema (2).

En el sistema social actual, se asocia a la vejez con la dependencia en distintas formas y ámbitos (familiar, social e institucional), y con la discapacidad funcional, ya sea física o mental. Prueba de ello, es la prevalencia de estos estereotipos negativos que el Instituto Nacional de Geriatría (INGER) reporta en México, y que, a pesar de la nueva realidad favorable del envejecimiento, aún se encuentran presentes en la sociedad. Así pues, estereotipos como que los adultos mayores tienen un deterioro en su memoria (73.1\%), se enojan fácilmente (65.1\%), son como niños (62.3\%) y su salud es mala (60.2\%), son algunos ejemplos de ello (3).

Existen diversos estudios a nivel internacional que fundamentan la prevalencia de elevados estereotipos negativos hacia el envejecimiento, llegando inclusive a niveles académicos superiores, como el caso de los programas educativos que involucran a las Ciencias de la Salud ${ }^{(4-6)}$.

En la sociedad mexicana, para la profesión de enfermería, el análisis de las dimensiones del instrumento del Cuestionario de Evaluación de Estereotipos Negativos hacia la Vejez (CENVE) en una muestra de 95 estudiantes, reveló mayor frecuencia de estereotipos negativos en la dimensión salud (65.3\%), lo que hace alusión al fuerte deterioro fisiológico, de los procesos cognitivos y las incapacidades. Seguida de la dimensión de carácter-personalidad (49.5\%), al referir que son como niños y también rígidos e inflexibles. Y, por último, la dimensión motivacional-social (36.8\%) menciona la falta de interés hacia el sexo y a las cosas en general, ser menos activos y tener menos amigos ${ }^{(7)}$.

De igual manera, una investigación realizada en estudiantes universitarios del área de Ciencias de la Salud (Medicina, Salud Pública y Geriatría), comprobó mediante el instrumento CENVE, que los estereotipos hacia la vejez son mayormente negativos, afectando principalmente a las dimensiones de salud y carácter-personalidad (8). Recientemente, se realizó un estudio sobre los estereotipos 
negativos presentes en estudiantes de la Licenciatura en Enfermería hacia el envejecimiento. De una muestra de 180 estudiantes de 20.39 años $(D E=3)$, del género femenino (77\%); los estereotipos negativos, según el CENVE, estuvieron presentes en un $52.8 \%$ de la muestra, siendo la dimensión más afectada la de carácter-personalidad (62.2\%), seguida de la dimensión motivacional-social (61.7\%). El puntaje total medio del CENVE fue de $35(D E=6.26, M d=36, M i ́ n=17$, Máx= 39) ${ }^{(9)}$.

Como lo muestra el marco empírico, la representación centrada en aspectos negativos hacia el envejecimiento, afecta también al personal de salud (profesionales, estudiantes y docentes), vinculando este proceso vital con la soledad, el abandono, la pérdida financiera, el alejarse de la familia, las pérdidas (trabajo, familia, pareja), lentitud funcional y cansancio, e ideas entorno a la cercanía de la muerte ${ }^{(10)}$. Esta situación genera un ciclo que intensifica los estereotipos negativos presentes en el adulto mayor, ya que las personas mayores tienden a tomar las imágenes negativas que se tienen sobre ellos y perpetuar sus propios estereotipos negativos, reforzando estas creencias a nivel micro (interpersonal, familiar o intergeneracional), y macro (institucional, laboral, social), lo que a su vez conlleva al maltrato y abuso a este sector poblacional (11).

La población envejecida sufre de múltiples consecuencias negativas en todas sus esferas individuales por culpa de los estereotipos, por lo que se necesita modificar la forma en que se percibe al adulto mayor hoy en día. Uno de los objetivos de la Organización Mundial de la Salud (OMS) es "echar abajo los estereotipos asociados a la vejez", para consolidar un óptimo desarrollo de esta población, de tal manera que pueda integrar su quehacer diario en la sociedad actual, y con la consecuente mejora de su calidad de vida ${ }^{(12)}$.

Una de las consecuencias que acarrean los estereotipos es el denominado "viejismo", que compromete actitudes y concepciones discriminatorias hacia los adultos mayores (13), lo que refuerza los datos de la OMS, donde se argumenta que podría haber más discriminación por razones de edad que por el sexo o raza, manifestándose ya sea por prejuicios, actitudes, prácticas o políticas que perpetúan estas creencias desvalorizantes, incluso en el sector salud (14).

Así pues, el propósito de este estudio fue analizar la presencia e intensidad de los estereotipos negativos hacia el envejecimiento entre los estudiantes de los diferentes programas educativos del área de la salud de una universidad pública de la región centro de México.

\section{METODOLOGÍA}

Se realizó un estudio con enfoque cuantitativo, de corte transversal, descriptivo y comparativo en el período del 6 de marzo de 2017 al 1 de febrero de 2018, en uno de los campus de una Universidad Pública del Estado de Guanajuato. La población estuvo conformada por estudiantes matriculados durante el periodo agosto-diciembre 2017 de los programas educativos del área de la salud, tomando como base la matrícula inscrita durante el periodo enero-junio 2017 ( $\mathrm{N}=$ 2,110 estudiantes). Se obtuvo el tamaño de muestra mediante la fórmula para poblaciones finitas de $n=326$ (IC 95\%, Error de 0.05), muestreo estratificado proporcional resultante de Psicología Clínica $(n=69)$, Nutrición $(n=62)$, Terapia Física y Rehabilitación $(n=57)$ y, Enfermería y Obstetricia $(n=136)$.

Se solicitó la participación voluntaria de los estudiantes, y se les entregó el consentimiento informado, cumpliendo con lo establecido en la Ley General de Salud en materia de Investigación para la Salud Título Quinto, Capítulo Único, Artículo 100, Fracción IV (15) y con las consideraciones éticas y los principios de la Declaración de Helsinki (beneficia, respeto a la dignidad humana y justicia) de la Asociación Médica Mundial para las Investigaciones Médicas en Seres Humanos, en su actualización de $2013{ }^{(16)}$. También se contó con la aprobación del Comité de Investigación de la División de Ciencias de la Salud e Ingenierías del Campus CelayaSalvatierra de la Universidad de Guanajuato, con el registro CIDSC-3102908.

Se entregó a cada participante el cuestionario de datos sociodemográficos y el instrumento CENVE (2005), el cual consta de 15 ítems en escala Likert de 4 puntos (1= Desacuerdo Total a 4= De Acuerdo Total), cuyo valor fluctúa entre 15 y 60. Las puntuaciones más altas indican un elevado grado de estereotipos negativos hacia la vejez, valorando tres dimensiones:

-Salud: Comprende las afirmaciones del deterioro físico y cognitivo, así como de sus discapacidades.

-Motivacional-Social: Son las creencias acerca de las carencias socioafectivas.

-Carácter-Personalidad: Se refiere a la rigidez del pensamiento y los problemas de labilidad emocional.

El Alfa de Cronbach reportado por cada dimensión en su construcción original, fue de 0.67, 0.64 y 0.66, respectivamente ${ }^{(17)}$

Se realizó el análisis con estadística descriptiva por distribución de frecuencias, porcentajes, mínimos, máximos, medidas de tendencia central y dispersión (media, mediana y desviación estándar) en el Programa de Estadística para las Ciencias Sociales (Statistics Program for Social Sciences, SPSS v.22). La variable de estudio se analizó mediante la prueba estadística Z para dos proporciones, para diferencias entre grupos.

\section{RESULTADOS}

En lo referente a las carreras, el $42.0 \%$ cursa el programa educativo de Enfermería y Obstetricia, el 21.3\% Psicología Clínica, el 19.1\% Nutrición, y el 17.6\% Terapia Física y Rehabilitación. El semestre predominante es el Cuarto (29.3\%), seguido del Primero (27.5\%), Segundo (26.2\%), Sexto (9.0\%) y Quinto (7.1\%).

Los resultados sociodemográficos indican que la edad media de los participantes es de $20.15 \pm 1.67$ años. Para los 
Tabla 1. Edad de los participantes por programa educativo

\begin{tabular}{|c|c|c|c|c|}
\hline \multirow{2}{*}{ Edad (años) } & \multicolumn{2}{|c|}{ Rango } & \multirow{2}{*}{$\bar{x}$} & \multirow{2}{*}{$D E$} \\
\hline & Mín & Máx & & \\
\hline Total & 17 & 28 & 20.15 & 1.67 \\
\hline Enfermería y Obstetricia & 17 & 27 & 20.33 & 1.78 \\
\hline Psicología Clínica & 18 & 25 & 19.97 & 1.35 \\
\hline Nutrición & 18 & 28 & 20.56 & 1.91 \\
\hline Terapia Física y Rehabilitación & 18 & 23 & 19.47 & 1.25 \\
\hline
\end{tabular}

$X=$ Media, $D E=$ Desviación Estóndar

Tabla 2. Distribución por Género

\begin{tabular}{lll}
\hline Género & $f$ & $\%$ \\
\hline Total & 65 & 20.1 \\
Masculino & 259 & 79.9 \\
Femenino & & \\
Enfermería y Obstetricia & 20 & 14.7 \\
Masculino & 116 & 85.3 \\
Femenino & & 29 \\
Psicología Clínica & 20 & 71 \\
Masculino & 49 & 27.4 \\
Femenino & & 72.6 \\
Nutrición & 17 & \\
Masculino & 45 & $\mathbf{n = 3 2 4}$ \\
Femenino & & \\
Terapia Física y Rehabilitación & & \\
Masculino & 49 & \\
Femenino & & \\
\hline Fuente: Cuestionario de Dotos Sociodemogróficos, 2017 & & \\
\hline
\end{tabular}


Tabla 3. Distribución de los Estereotipos Negativos hacia el Envejecimiento

\begin{tabular}{|c|c|c|c|c|c|c|c|c|}
\hline \multirow[t]{2}{*}{ Variable/ Dimensión } & \multicolumn{2}{|c|}{ CENVE } & \multicolumn{2}{|c|}{ Dimensión Salud } & \multicolumn{2}{|c|}{$\begin{array}{c}\text { Motivacional- } \\
\text { Social }\end{array}$} & \multicolumn{2}{|c|}{$\begin{array}{c}\text { Carácter- } \\
\text { Personalidad }\end{array}$} \\
\hline & $f$ & $\%$ & $f$ & $\%$ & $f$ & $\%$ & $f$ & $\%$ \\
\hline \multicolumn{9}{|l|}{ Total } \\
\hline Estereotipos Altos & 120 & 37 & 157 & 48.5 & 68 & 21 & 179 & 55.2 \\
\hline Estereotipos Bajos & 204 & 63 & 167 & 51.5 & 256 & 79 & 145 & 44.8 \\
\hline \multicolumn{9}{|c|}{ Enfermería y Obstetricia } \\
\hline Estereotipos Altos & 56 & 41.2 & 66 & 48.5 & 39 & 28.7 & 78 & 57.4 \\
\hline Estereotipos Bajos & 80 & 58.8 & 70 & 51.5 & 97 & 71.3 & 58 & 42.6 \\
\hline \multicolumn{9}{|l|}{ Psicología Clínica } \\
\hline Estereotipos Altos & 27 & 39.1 & 31 & 44.9 & 15 & 21.7 & 32 & 46.4 \\
\hline Estereotipos Bajos & 42 & 60.9 & 38 & 55.1 & 54 & 78.3 & 37 & 53.6 \\
\hline \multicolumn{9}{|l|}{ Nutrición } \\
\hline Estereotipos Altos & 22 & 35.5 & 31 & 50 & 10 & 16.1 & 38 & 61.3 \\
\hline Estereotipos Bajos & 40 & 64.5 & 31 & 50 & 52 & 83.9 & 24 & 38.7 \\
\hline \multicolumn{9}{|c|}{ Terapia Física y Rehabilitación } \\
\hline Estereotipos Altos & 15 & 26.3 & 29 & 50.9 & 4 & 7 & 31 & 54.4 \\
\hline Estereotipos Bajos & 42 & 73.7 & 28 & 49.1 & 53 & 93 & 26 & 45.6 \\
\hline
\end{tabular}

subgrupos, la edad media se muestra en la Tabla 1, donde los estudiantes del programa educativo de Nutrición tienen una edad media mayor que el resto $(20.56 \pm 1.91)$.

La mayoría de los participantes son del género femenino (79.9\%), siendo mayor la presencia de mujeres en los programas educativos de Terapia Física y Rehabilitación (86\%) y Enfermería (85.2\%) (Tabla 2).

El puntaje total del CENVE en la muestra de estudio fue de 36.24 \pm 4.96 , para la dimensión salud el puntaje fue de 12.14 \pm 1.90 , para motivacional-social fue de $10.99 \pm 2.05$, y para la dimensión carácter-personalidad el puntaje resultante fue de $12.83 \pm 2.11$.

La presencia de un elevado grado de creencia en los estereotipos negativos hacia el envejecimiento se encontró en un $37 \%$ de los casos, predominando los estereotipos negativos en la dimensión de carácter-personalidad, (55.2\%), la cual aborda aspectos como la regresión a la infancia por parte del adulto mayor, y el declive del estatus de adulto; seguida de la dimensión salud (48.5\%) abarcando afirmaciones acerca del deterioro físico y cognitivo así como el surgimiento de discapacidades; y finalmente, la dimensión motivacional-social (21\%), que se refiere a las carencias socioafectivas que sufre el adulto mayor (Tabla 3).

Finalmente, se realizó la prueba $Z$ para dos proporciones con la finalidad de encontrar diferencias estadísticamente significativas en la presencia de estereotipos negativos entre los diferentes programas educativos. Los resultados no mostraron diferencias estadísticamente significativas entre los grupos, sin embargo, la comparativa entre el programa educativo de Enfermería y Obstetricia y Terapia Física y Rehabilitación, mostró un resultado limítrofe $(Z=1.96, p=0.05)$ (Tabla 4), pudiéndose inferir que la presencia elevada de estereotipos negativos hacia el envejecimiento afecta más a los estudiantes programa educativo de Enfermería y Obstetricia en comparación con los de Terapia Física y Rehabilitación.

\section{DISCUSIÓN}

Los resultados de la media de edad se asemejan a estudios realizados en el contexto internacional ${ }^{(4,5)}$, donde la edad media oscila entre los 20 y 21 años; y en México, por lo encontrado por Carrillo-Cervantes et al. ${ }^{(9)}$, donde existe 
Tabla 4. Comparación de proporciones de estereotipos negativos altos por programa educativo

\begin{tabular}{lcc}
\hline Comparación & Z & Valor de $P$ \\
\hline Enfermería y Obstetricia contra Psicología Clínica & 0.29 & 0.77 \\
Enfermería y Obstetricia contra Nutrición & 0.76 & 0.45 \\
Enfermería y Obstetricia contra Terapia Física y Rehabilitación & 1.96 & 0.05 \\
Psicología Clínica contra Nutrición & 0.43 & 0.67 \\
Psicología Clínica contra Terapia Física y Rehabilitación & 1.52 & 0.13 \\
Nutrición contra Terapia Física y Rehabilitación & 1.08 & 0.28 \\
\hline
\end{tabular}

Fuente: Elaboración propia, 2017

$n=324$

mayor similitud. Sin embargo, la edad media fue menor en comparación a lo expuesto por estudios realizados en Centro y Sudamérica ${ }^{(6,18,19)}$, donde el promedio fue menor a 30 años, y en México ${ }^{(7,8)}$, donde la edad oscilaba entre los 22 años.

Las diferencias encontradas pueden deberse a factores demográficos o culturales de las regiones, ya que, en México, el promedio de edad para los estudiantes matriculados en el nivel superior está entre los 18 y 20 años según la Organización para la Cooperación y el Desarrollo Económico (OCDE), coincidiendo con los resultados obtenidos ${ }^{(20)}$.

Sin embargo, a pesar de la Reforma Educativa del 2012, aún se presenta un alto nivel de deserción en la educación superior del país, debido a que el contexto actual exige que el adulto joven pase más tiempo trabajando que estudiando, y por consecuencia, únicamente el 24\% de los jóvenes de 20 años en México están matriculados en educación superior, representando así un área de oportunidad educativa en el país (20).

Referente al género, existe similitud con investigaciones realizadas principalmente en Centro y Sudamérica ${ }^{(5,18,21)}$; así como por lo reportado por Durán-Badillo et al. (7), y CarrilloCervantes et al. ${ }^{(9)}$, en el país, donde el género femenino predominó en más del 75\% de los casos.

Sin embargo, se encontró diferencia entre poblaciones como España ${ }^{(4)}$ y Argentina ${ }^{(6)}$, donde los estudios mostraron que la presencia del género femenino es mayor del $85 \%$. Asimismo, el porcentaje obtenido en el presente estudio fue mayor en comparación con lo obtenido por León et al. (19), en Chile, y en el contexto nacional, por Domínguez et al. ${ }^{(8)}$, donde la presencia del género femenino fue menor del $70 \%$.

En México, todavía existe una predilección de esta población para elegir programas educativos que han sido socialmente considerados como "femeninos", como es el caso de aquellas pertenecientes al área de la salud (Enfermería, Nutrición, Psicología), donde por lo menos, un 75\% de la población que estudia en esta área, pertenece a este género (22). Ocurriendo lo mismo a nivel global, donde según datos de la OCDE, en el año 2013, las mujeres representaron el $78 \%$ de las nuevas inscripciones a programas educativos de nivel superior relacionados con la salud y el bienestar (23).

Posteriormente, se encontró que la presencia de estereotipos negativos hacia el envejecimiento fue menor que lo reportado en estudios de España ${ }^{(4)}$ y Costa Rica (24). En el primer caso, esta presencia representó el 62\% de la muestra, mientras que, en Costa Rica, el CENVE tuvo un puntaje medio de 51.52 .

Por el contrario, el puntaje del CENVE obtenido en el presente fue mayor que lo reportado por BlancoMolina y Pinazo-Hernandis (5) , y Portela ${ }^{(6)}$, donde el puntaje CENVE oscila entre los 32. De igual manera, la presencia de estereotipos negativos hacia el envejecimiento, fue mayor en contraste con estudios realizados en Chile y Argentina $(6,18,19)$.

En las dimensiones del CENVE, se encontró similitud con los resultados de Sequeira y Silva (18); mientras que en diversos estudios la dimensión más afectada por los estereotipos negativos fue la de Salud ${ }^{(4,6,21)}$.

Dentro del contexto nacional, la dimensión más afectada difiere según un par de estudios llevados a cabo en el noreste (7) y occidente ${ }^{(8)}$ del país, siendo en esos estudios, las dimensiones: Motivacional-Social y Salud, respectivamente.

Finalmente, existe similitud con una investigación reciente realizada en la zona norte de México ${ }^{(9)}$, cuya afectación reside en la dimensión Carácter-Personalidad; sin embargo, en el presente estudio el puntaje CENVE fue mayor, mientras que la presencia de elevados estereotipos negativos hacia el envejecimiento fue menor (37\% contra $52.8 \%)$. 


\section{CONCLUSIONES}

Actualmente, los elevados estereotipos negativos prevalecen en la población de estudio, donde la dimensión más afectada fue la de carácter-personalidad; "los mayores de 60 años chochean", "las personas mayores son como niños", y "las personas mayores se hacen rígidas e inflexibles", son algunos de los estereotipos dominantes. Así pues, se sugiere la inclusión de programas educativos enfocados a modificar los estereotipos negativos hacia el envejecimiento desde la formación básica.

Resulta importante puesto que en el Plan de Estudios que la Secretaría de Educación Pública (SEP) de 2011, dentro de las metas previstas están temáticas de relevancia social como la atención de la diversidad, considerando programas que impliquen la diversidad etaria, creencias sociales referentes al adulto mayor y el proceso de envejecimiento, ya que a pesar del cambio demográfico que está sufriendo el país, la relevancia a estos programas es escasa. Así se potenciará la construcción de habilidades socioemocionales o educación socioemocional en la población.

Básicamente, si este tipo de programas se implementan eficientemente desde edades tempranas, la perspectiva social que aqueja a la población adulta mayor podrá remitir paulatinamente, y a futuro, si una persona decide involucrarse como profesional de la salud, podrá contribuir con la disminución del viejismo ocasionado por los estereotipos, y, por consiguiente, el desarrollo de su profesión y la calidad en el servicio brindado a esta población se verán beneficiados.

\section{CONFLICTO DE INTERESES}

Los autores declaran no tener ningún conflicto de intereses.

\section{FINANCIAMIENTO}

Se agradece a la Universidad de Guanajuato campus CelayaSalvatierra sede Celaya por su colaboración con los recursos materiales para la realización del estudio.

\section{REFERENCIAS BIBLIOGRÁFICAS}

1. Instituto Nacional de Estadística y Geografía. Estadísticas a propósito del día internacional de las personas de edad [Internet]. Aguascalientes: INEGl; 2016 [citado 3/07/2017]. Disponible en: http://www.inegi.org.mx/saladeprensa/ aproposito/2016/edad2016_0.pdf

2. Gascón S. Imágenes negativas y estereotipos sobre la vejez, maltratos que inciden en las políticas de salud. I salud [Internet]. 2015 [citado 3/07/2017]; 10(47):6-10. Disponible en: www.isalud.edu.ar/news/links/isalud47.pdf
3. Instituto Nacional de Geriatría. Hechos y desafíos para un envejecimiento saludable en México [Internet]. México: INGER; 2016 [citado 3/07/2017]. Disponible en: http://www. geriatria.salud.gob.mx/descargas/noticias/hechos-desafios. pdf

4. Sarabia MC, Castanedo C. Modificación de estereotipos negativos en la vejez en estudiantes de enfermería. Gerokomos [internet]. 2015 [citado 19/01/2018]; 26 (1):1012. Disponible en: http://scielo.isciii.es/pdf/geroko/v26n1/ original2.pdf

5. Blanco-Molina M, Pinazo-Hernandis S. Información y exposición indirecta para reducir estereotipos hacia el envejecimiento. International Journal of Developmental and Educational Psychology [internet]. 2016 [citado 11/01/2018]; 1(2):367-80. Disponible en: https://doi.org/10.17060/ ijodaep.2016.n2.v1.707

6. Portela A. Estereotipos negativos sobre la vejez en estudiantes de Terapia Ocupacional. Revista Argentina de Terapia Ocupacional [internet]. Ago 2016 [citado 3/07/2017]; 2(1): 3-13. Disponible en: http://www.revista.terapiaocupacional.org.ar/descargas/volumen2/Rev2\%20Art1.pdf

7. Durán-Badillo T, Miranda-Posadas C, Cruz-Barrera LG, Martínez-Aguilar ML, Gutiérrez-Sánchez G, AguilarHernández RM. Estereotipos negativos sobre la vejez en estudiantes universitarios de enfermería. Rev Enferm Inst Mex Seguro Soc [internet]. 2016 [citado 3/07/2017]; 24 (3): 205-9. Disponible en: www.medigraphic.com/pdfs/enfermeriaimss/ eim-2016/eim163i.pdf

8. Domínguez W, Estrada MC, Ibarra DG, Villalobos MS, Mendoza NM. Ideas sobre la vejez en estudiantes de Ciencias de la Salud del Centro Universitario de Tonalá. Investigación y práctica en psicología del desarrollo [internet]. Ene 2015 [citado 3/07/2017]; 1 (1): 237-43. Disponible en: http:// revistas.psico-ags.net/index.php/ippd/article/view/20

9. Carrillo-Cervantes AL, Villarreal -Reyna MA, MorenoPérez NE, Vega-Macías D, Delabra-Salinas MM. Estereotipos negativos y ansiedad ante el envejecimiento en estudiantes de licenciatura en Enfermería. Revista Médica Electrónica PortalesMedicos.com [internet]. Sep 2017 [citado 11/01/2018]; 1-9. Disponible en: https://www.revista-portalesmedicos. com/revista-medica/estereotipos-negativos-ansiedad-anteel-envejecimiento/

10. Guerrero-Castañeda RF, Ojeda MG. El envejecimiento desde la percepción de enfermería. Revista Enfermería Actual de Costa Rica [internet]. Ene-Jun 2017 [citado 4/07/2017]; (32). Disponible en: http://dx.doi.org/10.15517/ revenf.v0i32.23401 
11. Orozco-Campos N, López-Hernández D. Viejismo y empoderamiento. Los prejuicios de la vejez y la visión del propio envejecimiento. REDNUTRICIÓN [internet]. 6 May 2016 [citado 4/07/2017]; 7(19):245-50. Disponible en: https:// www.researchgate.net/publication/301963351_Viejismo_y_ empoderamiento_Los_prejuicios_de_la_vejez_y_la_vision_ del_propio_envejecimiento_Ageism_and_empowerment_ Prejudices_of_old_age_and_vision_of_aging_itself

12. Organización Mundial de la Salud [Internet]. Geneva: OMS; 2017 [actualizada 2017; citado 4/07/2017]. Disponible en: http://www.who.int/ageing/about/fighting_stereotypes/ es/

13. Lorenzo MC. El viejismo discrimina. El Cactus [internet]. Nov 2015 [citado 4/07/2017]; 4(4):36. Disponible en: https:// revistas.unc.edu.ar/index.php/Cactus/article/view/13111

14. Organización Mundial de la Salud [Internet]. Geneva: OMS; 2017 [actualizada May 2017; citado 4/07/2017]. Disponible en: http://www.who.int/features/factfles/ageing/ es/

15. Ley General de Salud. Diario Oficial de la Federación [internet]. 2014 [citado 6/07/2017). Disponible en: https:// www.uv.mx/saisuv/files/2014/08/Ley-General-de-Salud.pdf

16. Asociación Médica Mundial [Internet]. Francia: AMM; 2013 [actualizada 5 May 2015; citado 6/07/2017]. Disponible en: $\quad$ https://www.wma.net/es/policies-post/declaracionde-helsinki-de-la-amm-principios-eticos-para-lasinvestigaciones-medicas-en-seres-humanos/

17. Díaz IF, Ramírez VM, Oropeza LE, González MA, Mendoza NM. Prevalencia de Estereotipos Negativos hacia los Adultos Mayores en Estudiantes de Odontología. Gerolnfo [internet]. 2016 [citado 5/07/2017]; 11(2):1-15. Disponible en: http://new. medigraphic.com/cgi-bin/resumen.cgi?IDARTICULO=70784

18. Sequeira D, Silva D. Estereotipos sobre la vejez en estudiantes y docentes de la Facultad de Ciencias de la Salud, Universidad UCINF. REVISTA AKADEMEIA [internet] Ago 2016 [citado 11/01/2018]; 7(1):103-19. Disponible en: http://revistas.ugm.cl/index.php/rakad/article/view/140

19. León S, Correa-Beltrán G, Giacaman R. Negative ageing stereotypes in students and faculty members from three health science schools. Gerodontology [internet]. 2015 [Citado 19/01/2018]; 32(2):141-8. Disponible en: http://dx.doi. org/10.1111/ger.12065

20. Organización para la Cooperación y el Desarrollo Económico. Panorama de la Educación 2014: Indicadores OCDE. México: OCDE; 2014 [Citado 19/01/2018]. Disponible en: http://www.oecd.org/mexico/TALIS-2013-country-noteMexico.pdf
21. López M, Sánchez J, Velásquez Y. Conocimientos que tienen los estudiantes de enfermería sobre estereotipos y su influencia en el adulto mayor. Repositorio Institucional RIUMA [internet] 11 Sep 2017 [citado 11/01/2018]:1-12. Disponible en: http://repositorio.unan.edu.ni/4229/

22. Corona T, Medina PO, Sarti EJ, Uribe P. La mujer y la salud en México. México: CONACYT. 2014. [citado 12/01/2018] ISBN 978-607-443-437-8. Disponible en: https://www. anmm.org.mx/publicaciones/CAnivANM150/L4-La-mujersalud-Mexico.pdf

23. Avvisati F. ¿A qué tipo de carrera científica aspiran los jóvenes de 15 años? PISA in Focus 2015 Results [internet] 2016 [citado 22/01/2018]; 1:1-6. Disponible en: http://educalab.es/ documents/10180/18220/pisainfocus69v4.pdf/67ba5e8e85d7-408b-a967-5fac85e5433f

24. Campos MA, Salgado E. Percepción sobre la tercera edad en estudiantes de primer nivel de la facultad de psicología de ULACIT y su relación con el desarrollo de competencias profesionales para el trabajo con adultos mayores. Revista Rhombus [internet]. Ene 2013 [citado 3/07/2017]; 10 (1): 1-30. Disponible en: http://www.ulacit.ac.cr/files/careers/104_ percepcion_de_estudiantes_sobre_tercera_edad.pdf 